\title{
Efficient energy for one node and multi-nodes of wireless body area network
}

Sondous Sulaiman Wali, Mohammed Najm Abdullah

Department of Computer Engineering, University of Technology, Baghdad, Iraq

\begin{tabular}{|c|c|}
\hline Article Info & ABSTRACT \\
\hline Article history: & Compression sensing approaches have been used extensively with the idea \\
\hline Received Feb 14, 2021 & $\begin{array}{l}\text { of overcoming the limitations of traditional sampling theory and applying } \\
\text { the concept of pressure during the sensing procedure. Great efforts have }\end{array}$ \\
\hline Revised Jul 16, 2021 & been made to develop methods that would allow data to be sampled in \\
\hline Accepted Aug 4, 2021 & $\begin{array}{l}\text { compressed form using a much smaller number of samples. Wireless body } \\
\text { area networks (WBANs) have been developed by researchers through the }\end{array}$ \\
\hline Keywords: & $\begin{array}{l}\text { creation of the network and the use of miniature equipment. Small structural } \\
\text { factors, low power consumption, scalable data rates from kilobits per second }\end{array}$ \\
\hline Castalia & to megabits per second, low cost, simple hardware deployment, and low \\
\hline Compressive sensing & $\begin{array}{l}\text { processing power are needed to hold the wireless sensor through lightweight, } \\
\text { implantable, and sharing communication tools wireless body area network }\end{array}$ \\
\hline Energy & Thus, the proposed system provides a brief idea of the use of WBAN using \\
\hline Healthcare system & IEEE 802.15 .4 with compression sensing technologies. To build a health \\
\hline Sensors & system that helps people maintain their health without going to the hospital \\
\hline Throughput & and get more efficient energy through compression sensing, more efficient \\
\hline Wireless body area network & $\begin{array}{l}\text { energy is obtained and thus helps the sensor battery last longer, and finally, } \\
\text { the proposed health system will be more efficient energy, less energy- } \\
\text { consuming, less expensive and more throughput. }\end{array}$ \\
\hline
\end{tabular}

This is an open access article under the CC BY-SA license.

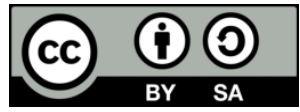

\section{Corresponding Author:}

Sondous Sulaiman Wali

Department Computer Engineering, The University of Technology

Al-Sinaa Street, Baghdad, Iraq

Email: ce.19.09@grad.uotechnology.edu.iq

\section{INTRODUCTION}

In the current era, health is the primary concern for every person. A significant number of doctors support the patient's day and night [1]. Patients suffering from primary diseases, such as diabetes, heartbeat, and high blood pressure, need frequent monitoring of their health status, resulting in a splurge of money. If the number of patients increases, a large number of physicians, hospitals, and other medical staff needed to attend will also need to increase [2]. Also, patients may need physical movement, which can be difficult, and due to day-to-day life, patients may no longer be required to stay in the hospital. As the health aspect is the main concern for all, many healthcare organizations thus emphasize the use of the wireless body area network (WBAN), e.g. the World Health Organization (WHO) [3]. With a growing interest in remote healthcare technologies, wireless body area sensor network (WBASN) gained considerable attention from both academic researchers and industry practitioners [4] in WBAN, the implanted sensors required energy to communicate with each other; thus, each sensor is fitted with a small battery. These devices are used to measure patient vital signs changes and relay data to the central node, which is responsible for organizing nodes and transmitting data to medical staff for examination and real-time diagnosis. However, the confidentiality and safety of patient records when exchanging medical information is a problem for WBAN [5]. Medical practitioners can electronically contact patient information through the internet, irrespective of 
patient location. The underlying goals of WBAN are the collection of physiological data from the patient(s) for continuous surveillance, which undoubtedly includes an effective routing approach [5], [6].

Reliable, secure, and effective implementation of the routing protocol is a challenge for WBAN due to its characteristics and drawbacks, such as energy reduction or overheating of embedded sensor nodes. Heat rise and energy loss affect the constancy of the network, so data is transmitted through different routes in WBAN. The various features of WBAN have increased the number of problems in a different layer of WBAN. The issues related to the medium-access control (MAC) layer are dynamic channel assignment, overhead packet management, overhead protocol, throughput, synchronization, and delay control. Network layer problems include versatility, location, traffic management, temperature and heat control, and optimal routing [7].

Some works are based on unique communication approaches like the cognitive cooperative to minimize power losses between nodes [8]. Other works used network encryption to minimize energy usage [9]. Some scholars confirmed that battery cell refinement is not enough tool to manage wireless body sensor network (WBSN) energy consumption [10]. While others some WBSN works are designed to save the sensor energies and minimize network data transmission [11]-[18]. Haseeb et al. [11] suggest a WBSN data transfer protocol that is energy efficient. As an input, the protocol proposes a set of network parameters including bandwidth, available sensor energies, and the number of hops to the coordinator, and the next-hop node, as an output, is chosen based on the weights and priorities of each parameter. Zhang and Yu [12] propose an energy-efficient WBSN mechanism based on a technique of sleep scheduling and a method of a set dominance. After the dominating graph was constructed, the sink selects a sub-set of datasets (e.g. active nodes) based on two approximate algorithms and a polymatroid function. Rabby et al. [13] a priority-based energy collection system was proposed for charging WBSN embedded sensor nodes. The scheme proposes to use the carrier sense multiple access with collision avoidance (CSMA/CA) protocol to transfer energy from the primary to the secondary device, thus minimizing sensor voltage and transmission losses.

The aim of this paper an appropriate health monitoring and decision-making system that facilitates close communication between patient and physician situations. The following can be included in our contributions to this paper: We designed and simulated a MAC protocol for a healthcare application and monitoring using IEEE 802.15.4 which includes the quality of service (QoS) required to access priority data to the channel; and added an algorithm to reduce the energy consumed when identifying and adapting a person's condition. The first goal is to alert the medical team if a prompt decision is reached. Then the sensor sends its data to the server to understand the individual's condition and send it to the required place (healthy, sick, and critical). This reduces the amount of periodic data that the sensor sends to save energy by eliminating redundancy. Finally, to demonstrate the performance of the algorithm and the energy-efficient by using simulators like Castalia to run simulations and compare results.

This paper consists of as follows: The description of the proposed technique is defined in section 2 . In section 3, a medical sensor is identified that is used in the proposed system. The proposed system is listed in section 4. Section 5 puts together the results and the different situations for several implementations of nodes in healthcare. The end of the work is noted in section 6.

\section{COMPRESSION SENSING TECHNIQUE}

Compressive sensing (CS) is a new field of study that has been widely established in recent years [19]. Strong mathematical foundations, defining the criteria for accurate signal reconstruction based on considerably fewer measurements (on Shannon's requirements) and proposals for a wide variety of solutions related primarily to the measurement matrix and conditions for reconstruction have opened a wide range of new possibilities and problems for many applications. The CS theory is based on two core principles: sparsity of signals and inconsistent sensing. The word "sparsity" means that the analyzed signal can be modeled with a limited number of components taken from a large dictionary. A signal can have either a sparse or compressible representation in the original domain or certain transform domains. Provided a poor signal, its information quality may be retrieved from what may seem to be an imperfect collection of measurements, at the cost of greater computational effort at the reconstruction level. The CS measurements are non-adaptive, i.e., they do not benefit from previous measurements. It can be processed or distributed efficiently because of fewer compressive measurements. CS makes a faithful reconstruction of the original signal from less random samples using certain non-linear reconstruction techniques. Because of all these features, CS finds its applications especially in areas where sensing is time-consuming or power-restricted [20].

For the reconstruction of the $\mathrm{x}$ sparse RN signal which may well be recovered only with the $\mathrm{M}<<\mathrm{N}$ component on the $\mathrm{M}^{*} \mathrm{~N}$ base matrix, compressive sensors are an alternative technique for the sampling of the Shannon/Nyquist [21]. For this reason, $\mathrm{x}$ must be sparse, i.e., k must be isolated from zero by $\mathrm{k}<<\mathrm{N}$. This technique is used to retrieve a sufficiently low signal from a small number of measurements [22]. 
CS is a method for calculating rare signals and then restoring these signals with missing results (in comparison to classical measurement methods). The method of data collection involves a comparatively limited amount of work. A limited number of measurements are carried out. Reconstruction is a dynamic process, but its execution is based on the analysis of a limited volume of data on the signal analyzed. The CS signal processing scheme involves both the acquisition and restoration versions as shown in Figure 1.

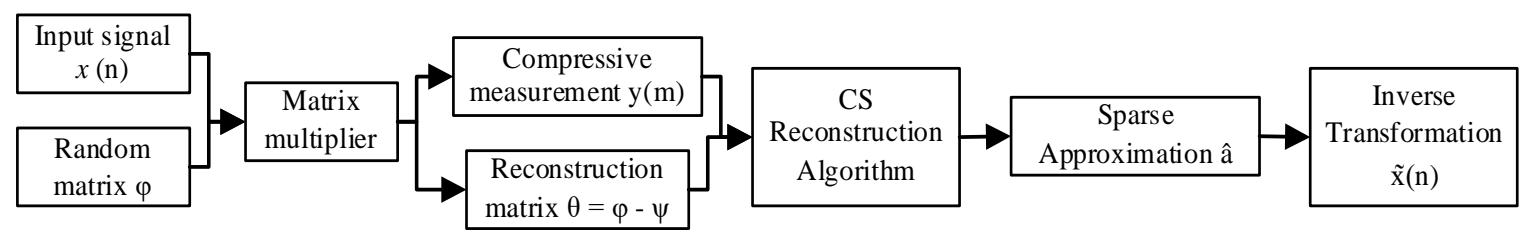

Figure 1. The method of signal processing is based on CS [23]

Essentially, the principle of CS includes three stages:

- The determination of the scarce decomposition of the signal,

- The configuration of the relevant compression representing the matrix, which closely approximates the initial signal of length $\mathrm{N}$ to the minimal $\mathrm{M}$ coefficients,

- Applying the corresponding reconstruction algorithm, which can recreate the initial signal from the observed M coefficients obtained from step 2.

\section{PROPOSED WORK}

Some of the problems with the proposed work in the WBAN shown in Figure 2 are reliability, scalability, and power management. Power management is a major problem. Layer phase physical and MAC optimization will reduce power consumption [24]. Due to its clear hybrid channel access mechanisms, we prefer IEEE 802.15.4 for experiments and implementation.

Castalia is a wireless sensor network (WSN), body area network (BAN) simulation model, and a standard computer network with low power. With the support of objective modular network testbed in C++ $(\mathrm{OMNeT++})$, use Castalia. The network is simulated with 1 node to 9 different nodes. Our patient has one or more sensors (sensors: electrocardiogram (ECG), temperature, skin temperature, blood pressure, PH, heart rate, Spo2, and motion sensor) Biomedical data routinely periods of varying data rates [25], [26]. The ECG magnetometer produces periodic data for high-temperature sensors, and generate low data annually. Therefore, all criteria must be fulfilled concurrently.

Using Castalia simulation, with a single/multiple sensors in each person in a node, to implement the idea, we increase or decrease the number of used nodes (the sensor) in the patient and then notice the effect of the packet reception rate, the packet loss rate, and the energy measured in $\mathrm{NJ} / \mathrm{bit}$. We note the simulation time reached and the number of responses received when the simulation stopped. In the end, we applied the idea to more than one patient, and here we used from five patients to 100 patients in the simulation noting the difference in results before and after using the compressive sensor technology.

In addition to the basic requirements of WBAN related to sensors and data transmission, the proposed WBAN design framework has some significant and requirements. The specification also requires the devices themselves and the network connection to the display. Some factors for optimizing a successful system are presented here:

- Data sensitivity: If not all WBAN applications are time or loss-sensitive. This is the case in applications relating to medical and sports. This requirement shall be met by multiplying points of access and a welldesigned MAC layer.

- Mobility: One of the key benefits of WBAN applications is to allow WBAN to function and, if necessary, exercise its activities. However, due to the distance and the signal reflection, this versatility of WBAN subjects may also lead to the disconnection from the access point. Then it is important to understand how communication is maintained and how networks are used when designing a WBAN that offers efficient energy.

- Adaptability: It is important to build a system that is capable of adjusting to any changes, such as adding or removing a sensor from/to the system or WBAN.

- Quality of service: Several parameters include delay, jotter, data loss, data rate [27]. 


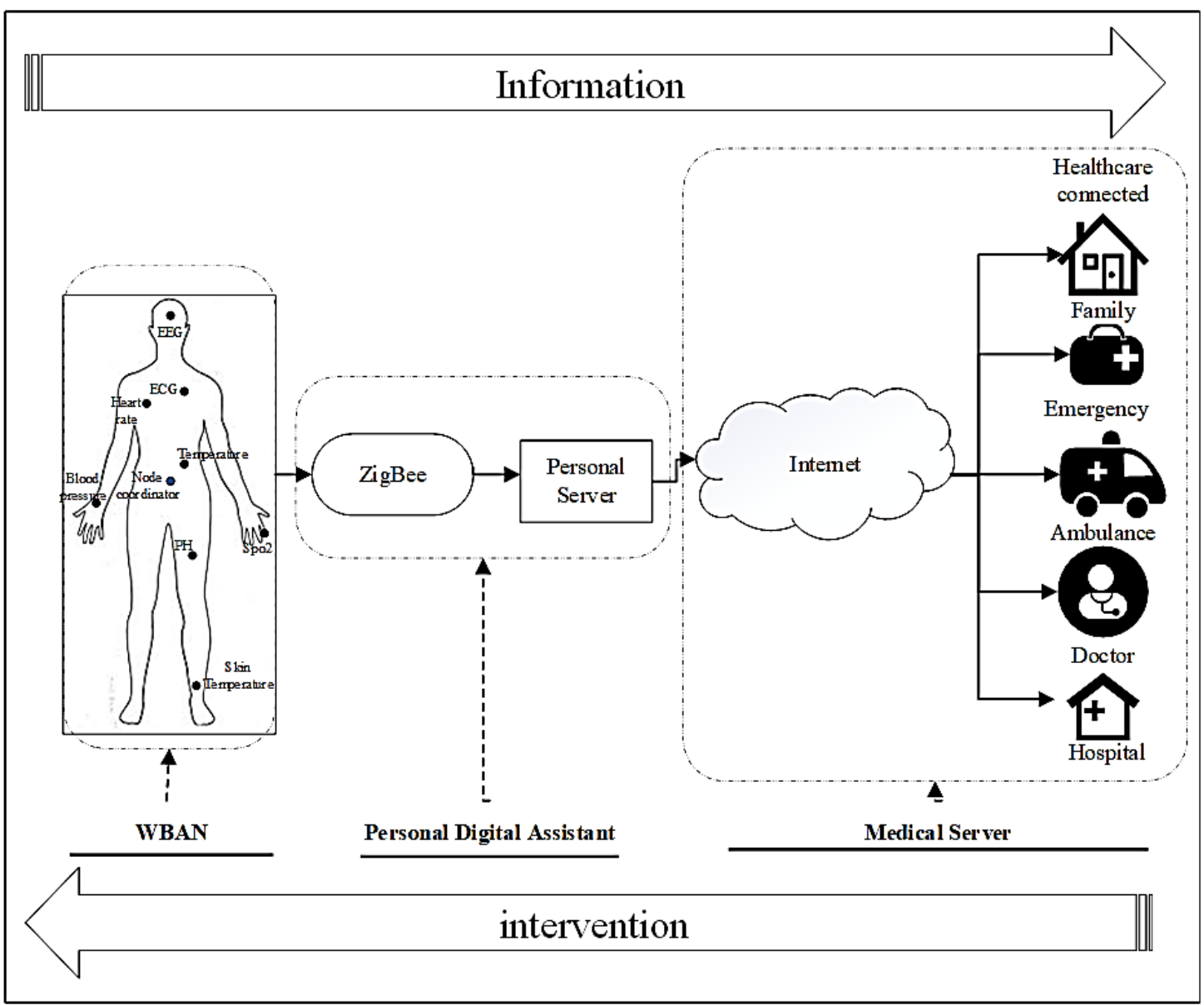

Figure 2. Block diagram of the proposed system

\subsection{Tiers architectures of the proposed system}

Data or knowledge is a cornerstone of the system like every other aspect depends upon, within the proposed WBAN system. The data generated by a WBAN is supposed to be used for the simulation. This information is usually not explicitly received by the user, it must be saved and analyzed or simply stored for future use. The information is however shown to the user before it is stored for real-time applications. Figure 2 shows how the system segments into different parts.

\subsubsection{Tier1: wireless body area network (WBAN)}

This part of the system constitutes the important part of the proposed system as each system depends on it. Any wearable sensor node is a source of knowledge from a person with a specific disease, from an athlete in practice, or an army/firefighter on the battlefield. It must be taken into account to reduce energy consumption, which is one of the main factors in WBAN. Therefore, we used the guaranteed time slots (GTS) protocol, which guarantees data delivery with the least possible amount of energy and to achieve the quality of medical service for the required applications.

\subsubsection{Tier2: personal digital assistant (PDA)}

In this part, the personal digital assistant is through this part that collects the physical data from the wearable sensors and sends the data to the remote station via one of the available networks (Wi-Fi, Bluetooth, ZigBee). In the proposed system ZigBee was used for future storage and processing that after it collects it and sends it to the personal server, which is like a database that stores and shares information. It is a proprietary server through which a person can control it and operate it to meet the needs of the person.

\subsubsection{Tier3: pedical server}

This part concerns the medical conditions of the person and records important improvements in the person's standard of life, and the medical server contains the following: i) doctors who treat and prescribe 
physiological parameters of patients, ii) emergency staff who intervene with patients in case of an accident, iii) home if the person does not suffer from any disease, iv) the hospital is in the case of the person who needs it, v) ambulance in the case of a person whose condition is an emergency.

This control unit provides access to the data in remote databases for viewing, reviewing, or editing any associated recommendations. In other words, the medical server communicates with the database. This three-tier architecture is an architecture based on information, i.e., data. This allows for WBAN versatility and remote storage in comparison to the legacy of proposed architectures, enabling the heterogeneity of the network and framework. It also makes it simple for various modules to communicate and allows for an abstraction of implementation. This is more advantageous because all WBAN systems can be built irrespective of the objective application with this architecture.

\subsection{Parameters metrics}

The specification and concept of routing protocols for WBANs face several challenges and limitations including throughput, power, autonomous management, radio, and protection. Below are some metrics used to measure the energy efficiency and throughput of WBANs.

\subsubsection{Throughput}

The throughput is the mean total traffic obtained correctly by the hub in each superstructure. Network throughput and node life provide insights for the wireless body sensor network of a large scale. The performance shall be seen as the bit amounts obtained by the time unit coordinator (second). The throughput was used with the IEEE 802.15.4 protocol, which is more reliable than IEEE 802.15.6. In low traffic conditions and the low latency provided by this protocol, it makes its use more efficient in WBAN in terms of throughput [25]. The equation below was used to determine the throughput from during the size of the packets and the time that the nodes pass (1):

$$
\text { Throughput }=\frac{\text { Packet Size }}{\text { DelayTime }}
$$

\subsubsection{Compressed data}

The compressed sensing data is used to reduce the sensing time and sampling rate and to obtain more energy efficiency. These high levels of information need to be sensed, such as obtaining a signal and then sending it, as in wireless and wireless communication systems, and then processing it. These tasks require data processing, storage memory, high bandwidth, and transmission, and the most important thing is more energy, especially the sensor, so the sensor was used. Compression as sparse high dimensional signals is obtained by taking necessary samples and avoiding oversampling [28]. This is done with the help of the sensor matrix, and then the original signal is recovered using inverse transformation as mentioned in Figure 1. The equation below shows the equation below is used to sense the compressed of the used packets (2):

$$
\text { Compressed Data }=\text { Packet size } * \text { CompressRatio }
$$

In the proposed system, work was done on one node and several nodes. The following is an explanation of how to use compression in one node and on several nodes, and we notice in the results the difference between them and how it affects throughput and efficient energy.

a. One node compressed sensing

One-node compressed sensing has four stages main: sparsity, sensing, quantization, and reconstruction signal. It can only handle small signals and needs the minimal isometry property (RIP). When the majority of its components are empty, a signal is known to be sparse. RIP demands that the matrix columns be distinct with a smaller size than the signal sparsity [29].

In the matrix of the sensing, the signal $x \in R^{N}$ is multiplied by a matrix $A \in R^{N * M}$ the signal to be received as with the classic compressive sensing. Where the $x \in R^{N}$ denotes the original signal and the $A \in$ $R^{N * M}$ denotes the sensing matrix. By observing (3), the multiplication among each quantized calculation must always be positive.

$$
A x_{i} * \operatorname{sign}\left(A_{i} x\right) \geq 0
$$

In the reconstruction part, to recover the signal from only the measurement signs [28]. To recover to the original signal by using the undetermined system by (4):

$$
\tilde{x}=\arg _{Y} \min \|x\|_{1} \text { s.t } Y=A x \geq 0
$$


where $\tilde{x}$ represents a reconstruction of the signal, and $Y=A x$ is noisy measurement models. Norm L1 was used instead of norm L0 or norm L2 because in norm L1 the closest sporadic point in the constraint line $x(n)$ was found. The compressed signal can be expressed as in noisy measurement models.

b. Multi-node compressed sensing

Multi-node compressed sensing has three stages main: sparsity, sensing, and reconstruction. In the sparsity to make a signal of high dimensions the signal $x \in R^{N}$ to render it sparse, it is viewed on a particular basis. The sparse signal is obtained in the sensing matrix stage by multiplying it by a sensing matrix, only to extract $\mathrm{M}$ measurements in $\mathrm{N}$, where $\mathrm{M}$ is smaller than $\mathrm{N}$. This multiplication can be seen as the signal projection into a base, also known as the dictionary, to hold the critical signal details only. As mentioned in the one node in the sensing stage. In the reconstruction process, the signal can be reconstructed from a few measures using a reconstruction algorithm at the receiver by estimating its coefficients [29]. This algorithm acts as a problem of optimization by solving the undetermined system (5):

$$
\tilde{x}=\arg _{R=A X+e} \min \|x\|_{1} \text { s.t } R=A X+e
$$

where $\tilde{x}$ represents reconstruction signal. Among other aspects, it constitutes the sparsest solution. And $R=A X+e$ represents noisy measurement models.

\subsubsection{Efficient energy}

Energy refers to the battery power used for any of the sensor nodes in the network [30]. Where the total power refers to the transmitted and received, and to the size of the transmitted and received packets, as shown in the equation below. As the energy is consumed through different sensing, encoding, transmitting, and receiving processes that are performed at any node of the sensor. A variety of efforts is being made to reduce the energy use of the sensor nodes in their treatment to increase the life of the network, make the network stable, and use compression sensing to obtain more efficient energy.

$$
\text { Energy }=\text { TxPower } * \text { SentPacketSize }+ \text { RxPower } * \text { ReceivedPacketSize }
$$

The following hypotheses are used during the simulation under Phase (I): i) the node is still actively attempting to give a packet, ii) ideal conditions for the channel, iii) the error rate of zero bit (BER), and iv) multi or one sender and one receiver. The simulation parameters are shown in Table 1. Simulation testing was performed on the Ubuntu16 machine with Castalia 3.3 operating under OMNeT++ 4.6. To assess the output before and after the use of the compression sensor technology.

Table 1. Simulation parameters

\begin{tabular}{cc}
\hline Parameters & Value \\
\hline Number of nodes & Varies 1-9 \\
Simulation area & $30 * 30$ \\
Frequency band & $2.4 \mathrm{GHz}$ \\
MAC & IEEE 802.15 .4 \\
Data rate & $1024 \mathrm{kbps}$ \\
Simulation time & $51 \mathrm{sec}$ \\
Channel mode & Lognormal wireless model \\
Physical bit per symbol & $2 \mathrm{bytes}$ \\
Transmission power & $15 \mathrm{dBm}$ \\
Physical packet overhead & $5 \mathrm{bytes}$ \\
MAC packet overhead & 7 bytes \\
Buffer size & 48 bytes \\
Performance parameters & Packet reception rate, packet loss rate, and Energy, Throughput \\
\hline
\end{tabular}

\section{RESULT AND DISCUSSION}

The simulation scenario is a MAC protocol-based BAN. Let's check the efficiency and the conditions of the wireless channel under different MAC functions. Check the packet distribution proportion under different MACs. Four scenarios were developed with the Castalia simulation: section 4.1 represents two scenarios in one node before and after sensing compression, in section 4.2 multi-node scenario before compression sensing, and section 4.3 multi-node scenario after sensing compression.

\subsection{One node without and with compression sensing technique}

In this section, one node in one or more patients, the scenario does not affect the nJ/bit energy as shown in Figure 3. No matter how many people there are. We also notice in Figure 3 that one node, whether 
before or after the compression sensing, regardless of the number of people, the energy is not affected, and in Figure 4 we notice the greater the number of people, throughput is increasing and that the rate of throughput is measured in kilobits per second (kbps) where the symbol $\mathrm{k}$ symbolizes the kilo, and the symbol $\mathrm{T}$ symbolizes time the determinant of the proposed system in the simulation.

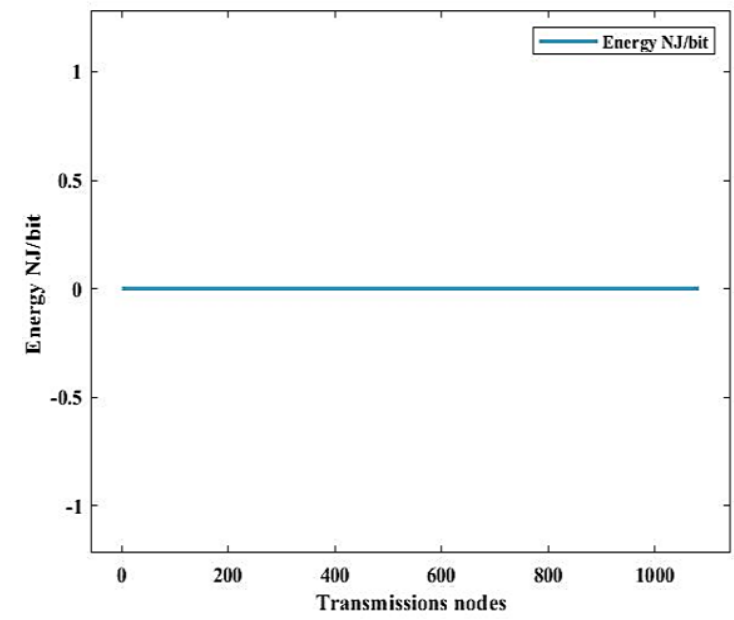

Figure 3. Effect of energy in one node

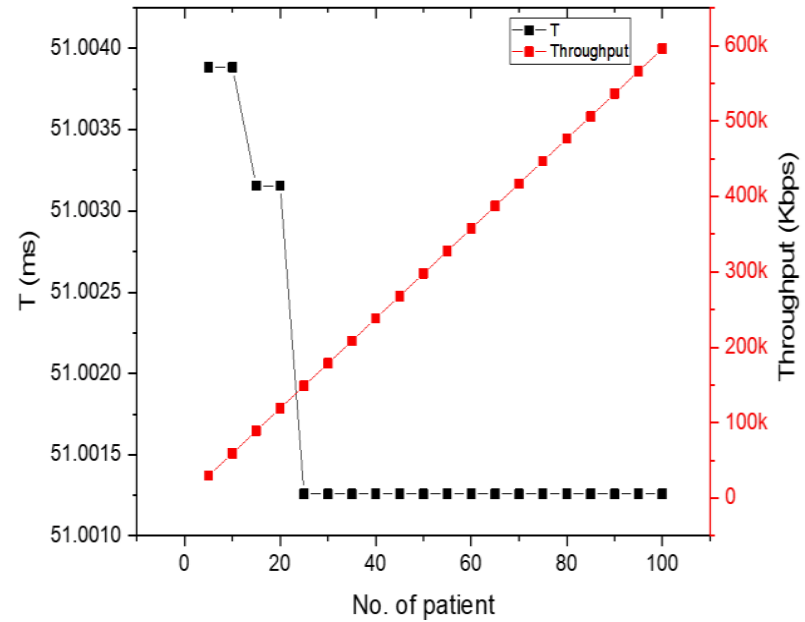

Figure 4. Throughput of one node

\subsection{Multi nodes without compression sensing technique}

In this section, a multi-node scenario before compressed affects one or more patients. In the proposed system there are multiple people in each person multiple nodes are observed when observing the packet reception rate, the packet loss rate, and the $\mathrm{nJ} / \mathrm{bit}$ energy. Where the energy rate is low, the packet received rate is also low, and there are losses in the packets, as we can see in Figure 5 the rate of energy, the rate of the received packets, and the losses in the packets, i.e., the nodes that were received and the nodes that were lost during the transmission.

\subsection{Multi nodes with compression sensing technique}

In this section, a multi-node compressed scenario affecting one or more patients. In the proposed system, there are multiple people per person. Multiple nodes are observed when monitoring packet reception rate, packet loss rate, and energy $\mathrm{nJ} / \mathrm{bit}$. Where we note in this section that by using compressed, the rate of packet loss is greatly reduced, and we note that the energy efficiency increases. As it indicates that a lot of nodes are received greatly using compression, as the loss of nodes is reduced. We also see in Figure 6 the energy rate, the received packet rate, and the packet losses.

WBANs ensure that people's lives are optimized. While the use of body networks in many areas of daily life was estimated to be a leading factor, the use of such structures is now very limited [31]. In Figure 7, it is observed that the energy, packet reception rate, and packet loss rate at a single node are not affected before or after the compressed sensor. But the energy reaches a minimum of $205.674 \mathrm{NJ} / \mathrm{bit}$, and a maximum of $228.134 \mathrm{NJ} / \mathrm{bit}$ before the compressed is sensed in the many nodes, but when the compressed is turned on, the energy will increase from the minimum of $390.981 \mathrm{NJ} / \mathrm{bit}$ to the maximum $433.082 \mathrm{NJ} / \mathrm{bit}$ in the many nodes and the proof of this is that the energy remains in preserving its efficiency after sensing the compressed, and thus the battery does not deplete quickly. And through receiving patients we conducted many experiments on the simulation used here which is Castalia, and in this figure, the energy in one node and several nodes were compared before and after the compressed sensing.

We notice that throughput increases in multiple nodes, as the symbol $\mathrm{M}$ refers to Mega, which indicates an increase in throughput in many nodes, whether before or after compression sensing, although throughput is measured in kilobits per second, as shown in Figure 8. The throughput per node and several nodes are compared, noting the time used in the simulation, as shown in Figure 9. Four scenarios were worked out in our proposed system to obtain more efficient energy and more throughput, as 802.15.4 is widely used as a radio standard for low-power radios and frequency bands that use $2.4 \mathrm{GHz}$, which provides a high analysis of the single node and multiple nodes, which has a high impact on throughput, and by using compressed sensing technology, more efficient and throughput energy was obtained. 


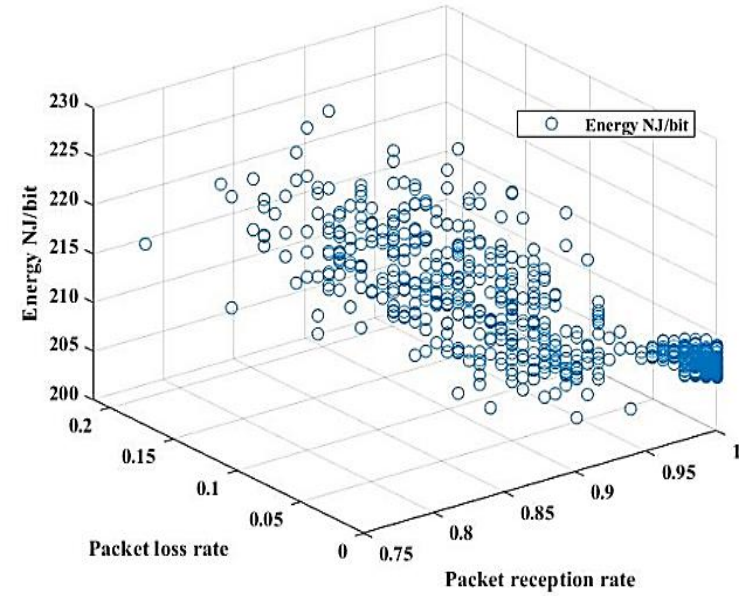

Figure 5. Multi-node without compressed

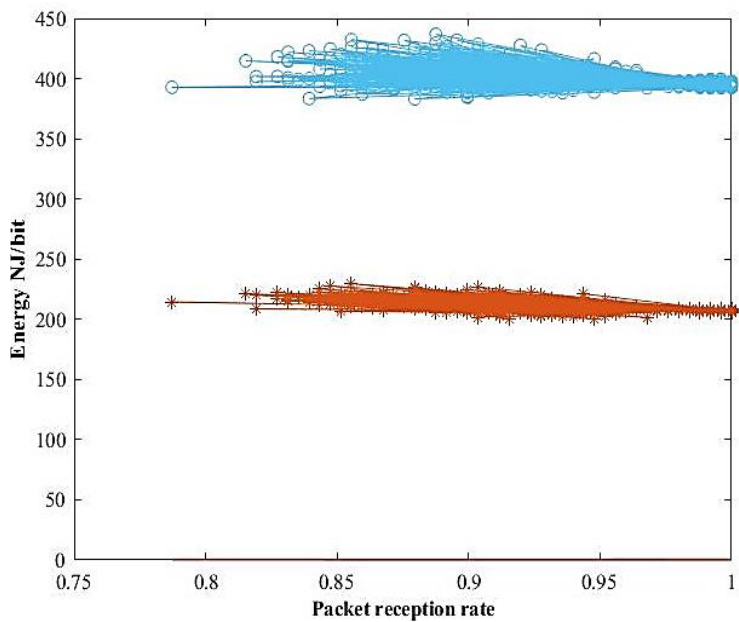

Figure 7. Compare energy in multi-node without and with compressed

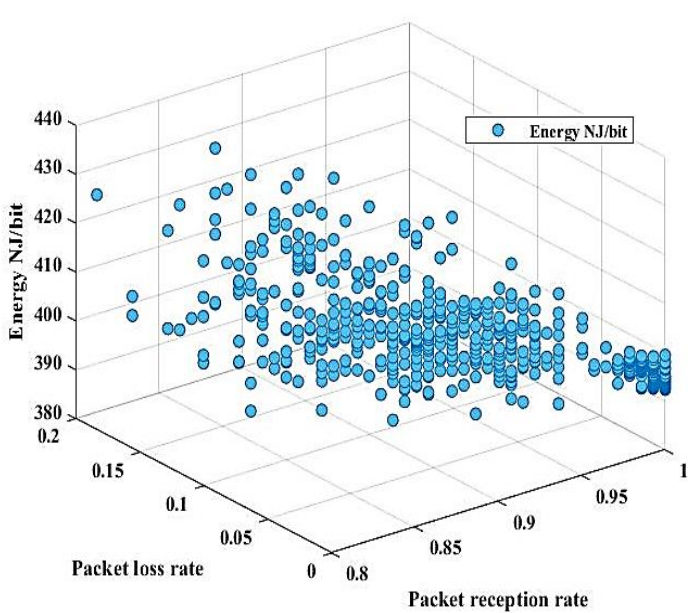

Figure 6. Multi-node with compressed

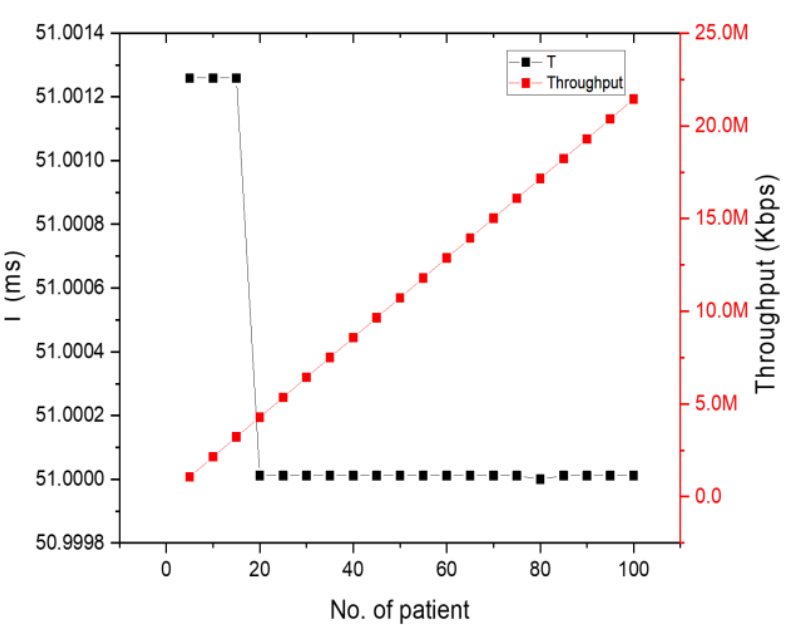

Figure 8. Throughput of multi-node

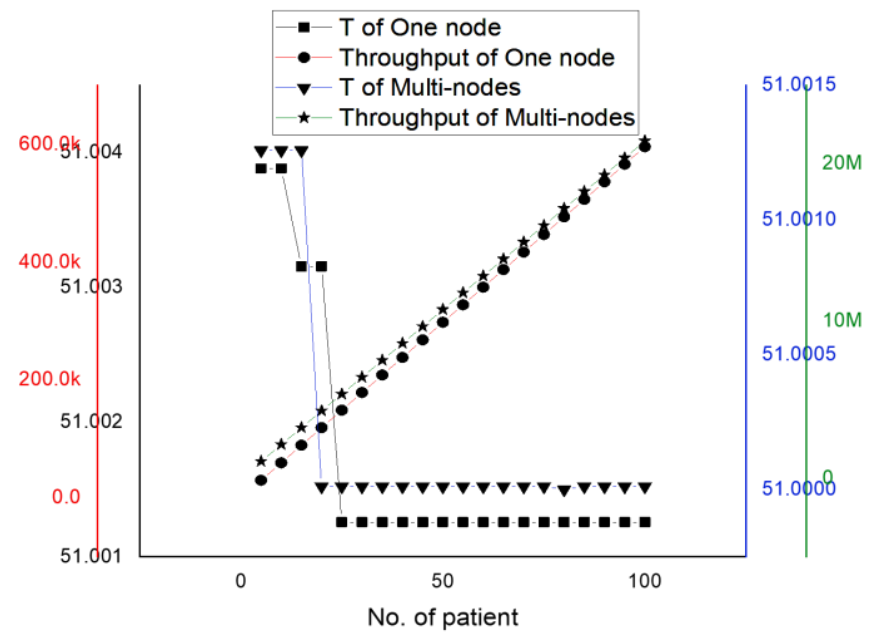

Figure 9. Comparison throughput of one and multi-node 


\subsection{Comparison with existing work}

This analysis contrasts deep with differing packet rates for single/multi-node nodes to accommodate both normal and contingencies. Other set work has targeted rate packets for all nodes that may differ equally without regard to priority for coping with emergencies. To increase the scalability of the design. They have been interacting with several nodes. This work's objectives. A workable WBAN situation with the capacity to manage a situation of any sort. In terms of QoS and simulation, compared with the corresponding job, the technologies used are different, as shown in Table 2.

Table 2. Comparison with the existing work

\begin{tabular}{ccccccc}
\hline Authors & Hybrid & Simulator & Medical & CS & Energy efficiency & Year \\
\hline Akbar et al. [26] & $\checkmark$ & $\checkmark$ & $\checkmark$ & $\mathbf{x}$ & $\checkmark$ & 2017 \\
Amin et al. [32] & $\checkmark$ & $\checkmark$ & $\checkmark$ & $\mathbf{x}$ & $\checkmark$ & 2017 \\
Navya et al. [33] & $\checkmark$ & $\checkmark$ & $\checkmark$ & $\mathbf{x}$ & $\checkmark$ & 2017 \\
Gupta and Biswas [34] & $\checkmark$ & $\checkmark$ & $\checkmark$ & $\mathbf{x}$ & $\checkmark$ & 2019 \\
Implemented & $\checkmark$ & $\checkmark$ & $\checkmark$ & $\checkmark$ & $\checkmark$ & 2021 \\
\hline
\end{tabular}

In this work, the design features are all related to the compressed sensing (CS) technology used for signal acquisition that benefits from a lower power consumption as illustrated in Figure 5, the packet loss rate is reduced by compressor technology and the packet reception is good. The packet rate of the single/multinode is based on the sensors in our case and the allocation rate will depend on the sensor's packet transfer rate and the emergency-related priority so that the obtained results exceed the current function.

\section{CONCLUSION}

In this paper, we discuss WBAN using OMNeT with Castalia simulation. This was done via one of the MAC protocols which is IEEE 802.5.14 on the data link layer using some compression sensing techniques that constantly sampling devices, pre-processing, processing, and transferring data, which leads to increased battery usage. The battery is also too small to prevent people from using the device from causing any pain. In this relationship, the emergence of CS has challenged established signal processing techniques, such as the popular sampling theory of Nyquist-Shannon, which states that sampling frequencies can be used at frequencies lower than those of Nyquist. In some areas, this finding is very important. The amount of data to be sampled must be reduced, and the amount of data to be transmitted should be reduced. In contrast, the battery consumption decreased, and the energy efficiency increased. Where it is increased from $70 \%$ to $90 \%$. That is, it increased by $20 \%$. By identifying the need for WBSN over wired networks, good results were obtained as more energy efficiency and increased throughput were obtained. Also, compare the results before and after using the compressed sensing technology. We believe that this study helps in developing better MAC protocols and guidelines for different medical applications of protocol designers and researchers. We believe compression sensing technologies are effective in WBAN. In future work, the implementation of hardware for the proposed system helps a person maintain his health.

\section{REFERENCES}

[1] H. Qadri, R. Ahmad, B. Mohammad, A. Mehmood, and G. Hamid, "Fresh water pollution dynamics, and remediation," SpringerLink, Springer Nature Singapore Pte Ltd., 2020, doi:10.1007/978-981-13-8277-2.

[2] F. Jamil, S. Ahmad, N. Iqbal, and D. H. Kim, "Towards a remote monitoring of patient vital signs based on iot-based blockchain integrity management platforms in smart hospitals," Sensors, vol. 20, no. 8, 2020, Art. no. 2195, doi: 10.3390/s20082195.

[3] K. Hasan, K. Biswas, K. Ahmed, N. S. Nafi, and M. S. Islam, “A comprehensive review of wireless body area network," Journal of Network and Computer Applications, vol. 143, pp. 178-198, 2019, doi: 10.1016/j.jnca.2019.06.016.

[4] M. Shu, D. Yuan, C. Zhang, Y. Wang, and C. Chen, "A MAC protocol for medical monitoring applications of wireless body area networks," Sensors, vol. 15, no. 6, pp. 12906-12931, 2015, doi: 10.3390/s150612906.

[5] W. D. Patel, S. Pandya, B. Koyuncu, B. Ramani, S. Bhaskar, and H. Ghayvat, "NXTGeUH: Lorawan based next generation ubiquitous healthcare system for vital signs monitoring falls detection," 2018 IEEE Punecon, 2018, pp. 1-8, doi: 10.1109/PUNECON.2018.8745431.

[6] O. N. Al-Khayat, S. Y. Ameen, and M. N. Abdallah, "WSNs power consumption reduction using clustering and multiple access techniques," International Journal of Computer Applications, vol. 87, no. 9, pp. 33-39, 2014, doi: 10.5120/15239-3780.

[7] Y. Qu, G. Zheng, H. Ma, X. Wang, B. Ji, and H. Wu, "A survey of routing protocols in WBAN for healthcare applications," Sensors, vol. 19, no. 7, 2019, doi: 10.3390/s19071638.

[8] A. Alkhayyat, A. A. Thabit, F. A. Al-Mayali, and Q. H. Abbasi, "WBSN in IoT health-based application: Toward delay and energy consumption minimization," Journal of Sensors, vol. 2019, 2019, Art. no. 2508452, doi: 10.1155/2019/2508452.

[9] A. Anwar and S. Duraisamy, "A predictive routing algorithm for WBSN based on Kalman filter iterations," IEEE Sensors Journal, vol. 18, no. 18, pp. 7741-7748, 2018, doi: 10.1109/JSEN.2018.2847049. 
[10] B. Sadiq, E. A. Adedokun, and Z. M. Abubakar, "The impact of mobility model in the optimal placement of sensor nodes in wireless body sensor network," 2017 IEEE 3rd International Conference on Electro-Technology for National Development (NIGERCON), 2017, pp. 702-705.

[11] K. Haseeb, A. Almogren, N. Islam, I. U. Din, and Z. Jan, “An energy-efficient and secure routing protocol for intrusion avoidance in IoT-based WSN," Energies, vol. 12, no. 21, 2019, Art. no. 4174, doi: 10.3390/en12214174.

[12] R. Zhang and J. Yu, "Energy-efficient algorithms and protocols for wireless body sensor networks," Springer International Publishing, 2020, doi: 10.1007/978-3-030-28580-7.

[13] M. K. M. Rabby, M. S. Alam, and M. S. T. S. A. Shawkat, "A priority-based energy harvesting scheme for charging embedded sensor nodes in wireless body area networks," PLoS One, vol. 14, no. 4, pp. 1-22, 2019, Art. no. e0214716, doi: 10.1371/journal.pone.0214716.

[14] R. A. Khan, Q. Xin, and N. Roshan, "RK-energy efficient routing protocol for wireless body area sensor networks," Wireless Personal Communications, vol. 116, no. 1, pp. 709-721, 2021, doi: 10.1007/s11277-020-07734-z.

[15] A. H. Sodhro, S. Pirbhulal, M. M. Lodro, and M. A. Shah, "Chapter 16: Energy-efficiency in wireless body sensor networks," Networks of the Future, Chapman and Hall/CRC, pp. 339-354, 2018.

[16] N. Geddes, G. S. Gupta, and F. Hasan, "An energy-efficient protocol for wireless body area network of health sensors," 2019 IEEE International Instrumentation and Measurement Technology Conference (I2MTC), 2019, pp. 1-6, doi: 10.1109/I2MTC.2019.8826862.

[17] A. Nahali, A. Hamdi, M. Gautier, A. Courtay, and R. Braham, "Energy modeling of wireless body area networks with on-body communication channel characterization," 2019 15th International Wireless Communications and Mobile Computing Conference (IWCMC), 2019, pp. 220-225, doi: 10.1109/IWCMC.2019.8766608.

[18] L. Li and D. Li, “An energy-balanced routing protocol for a wireless sensor network,” Journal of Sensors, vol. 2018, 2018, Art. no. 8505616, doi: 10.1155/2018/8505616.

[19] E. Sejdić, I. Orović, and S. Stanković, "Compressive sensing meet time-frequency: An overview of recent advances in timefrequency processing of sparse signals,” Digital Signal Processing, vol. 77, pp. 22-35, 2018, doi: 10.1016/j.dsp.2017.07.016.

[20] B. Palczynska, "Signal reconstruction from sparse measurements using compressive sensing technique," International Workshop on Modeling Social Media, vol. 548, 2019, pp. 239-247, doi: 10.1007/978-3-030-11187-8_20.

[21] L. P. Guo, C. W. Kok, H. C. So, and W. S. Tam, "Two-band signal reconstruction from periodic nonuniform samples," 2019 27th European Signal Processing Conference (EUSIPCO), 2019, pp. 1-5, doi: 10.23919/EUSIPCO.2019.8902554.

[22] M. Ruiz and I. Montalvo, "Electrical faults signals restoring based on compressed sensing techniques," Energies, vol. 13, no. 8, 2020, Art. no. 2121, doi: 10.3390/en13082121.

[23] M. Rani, S. B. Dhok, and R. B. Deshmukh, "A systematic review of compressive sensing: Concepts, implementations, and applications," IEEE Access, vol. 6, pp. 4875-4894, 2018, doi: 10.1109/ACCESS.2018.2793851.

[24] C. Sukanya, K. Priya, V. Paul, and P. Sankaranarayanan, "Integration of wireless sensor networks and mobile cloud-A survey," International Journal of Computer Science and Information Technologies, vol. 6, no. 1, pp. 159-63, 2015.

[25] M. S. Akbar, H. Yu, and S. Cang, "Delay, reliability, and throughput based QoS profile: A MAC layer performance optimization mechanism for biomedical applications in wireless body area sensor networks," Journal of Sensors, vol. 2016, 2016, Art. no. 7170943, doi: 10.1155/2016/7170943.

[26] M. S. Akbar, H. Yu, and S. Cang, "IEEE 802.15.4 frame aggregation enhancement to provide high performance in life-critical patient monitoring systems," Sensors, vol. 17, no. 2, 2017, Art. no. 241, doi: 10.3390/s17020241.

[27] M. T. Arefin, M. H. Ali, and A. K. M. F. Haque, "Wireless body area network: An overview and various applications," Journal of Computer and Communications, vol. 05, no. 07, pp. 53-64, 2017, doi: 10.4236/jcc.2017.57006.

[28] F. Salahdine, N. Kaabouch, and H. El Ghazi, "One-bit compressive sensing vs. multi-bit compressive sensing for cognitive radio networks," 2018 IEEE International Conference on Industrial Technology (ICIT), no. 1, 2018, pp. 1610-1615, doi: 10.1109/ICIT.2018.8352422.

[29] S. K. Sharma, E. Lagunas, S. Chatzinotas, and B. Ottersten, "Application of compressive sensing in cognitive radio communications: A survey," IEEE Communications Surveys and Tutorials, vol. 18, no. 3, pp. 1838-1860, 2016, doi: 10.1109/COMST.2016.2524443.

[30] A. Ahmad and Z. Hanzalek, "An energy-efficient schedule for IEEE 802.15.4/ZigBee cluster tree WSN with multiple collision domains and period crossing constraint," IEEE Transactions on Industrial Informatics, vol. 14, no. 1, pp. 12-23, 2018, doi: 10.1109/TII.2017.2725907.

[31] S. F. Raza, C. Naveen, V. R. Satpute, and A. G. Keskar, "A proficient chaos-based security algorithm for emergency response in WBAN system,” 2016 IEEE Students' Technology Symposium (TechSym), 2016, pp. 18-23, doi: 10.1109/TechSym.2016.7872648.

[32] B. Amin, N. Ullah, S. Ahmed, M. Taqi, and A. Hanan, "Path-loss and energy efficient model (PLEEM) for wireless body area networks (WBANs)," 2017 International Symposium on Wireless Systems and Networks (ISWSN), 2017, pp. 1-6, doi: 10.1109/ISWSN.2017.8250018.

[33] V. Navya and P. Deepalakshmi, "Increased throughput to sink using multihop routing protocol for link efficiency in wireless body area networks (M-TSIMPLE)," 2017 IEEE International Conference on Intelligent Techniques in Control, Optimization and Signal Processing (INCOS), 2017, pp. 1-7, doi: 10.1109/ITCOSP.2017.8303107.

[34] R. Gupta and S. Biswas, "Priority-based IEEE 802.15.4 MAC by varying GTS to satisfy heterogeneous traffic in healthcare application," Wireless Networks, vol. 26, no. 3, pp. 2287-2304, 2020, doi: 10.1007/s11276-019-02149-6. 